\title{
Fragmentation modeling of a resin bonded sand
}

\author{
William Hilth ${ }^{1, \star}$ and David Ryckelynck ${ }^{1, \star \star}$ \\ ${ }^{1}$ Mines ParisTech, PSL Research University, MAT - Centre des Matériaux, \\ CNRS UMR 7633, BP 8791003 Evry, France
}

\begin{abstract}
Cemented sands exhibit a complex mechanical behavior that can lead to sophisticated models, with numerous parameters without real physical meaning. However, using a rather simple generalized critical state bonded soil model has proven to be a relevant compromise between an easy calibration and good results. The constitutive model formulation considers a non-associated elasto-plastic formulation within the critical state framework.

The calibration procedure, using standard laboratory tests, is complemented by the study of an uniaxial compression test observed by tomography. Using finite elements simulations, this test is simulated considering a non-homogeneous 3D media. The tomography of compression sample gives access to 3D displacement fields by using image correlation techniques. Unfortunately these fields have missing experimental data because of the low resolution of correlations for low displacement magnitudes. We propose a recovery method that reconstructs 3D full displacement fields and 2D boundary displacement fields. These fields are mandatory for the calibration of the constitutive parameters by using 3D finite element simulations. The proposed recovery technique is based on a singular value decomposition of available experimental data. This calibration protocol enables an accurate prediction of the fragmentation of the specimen.
\end{abstract}

\section{Introduction}

In casting foundry, resin bonded sand is used in the casting mold to create the internal cavities of cylinder heads for instance. After the solidification, the casting sand has to be extracted. This decoring operation is most commonly carried out by mechanical means, knocking on the part with pneumatic hammers to fracture the cores first, and then vibrating to eliminate the fragmented pieces. Our main aim is to develop a mechanical model able to anticipate the fragmentation of the cemented sand. Nevertheless, this model has to be simple enough to be easily calibrated and used in finite elements simulations.

The literature concerning resin bonded sand is limited and mostly consists in industrial studies varying the resin or its amount, or the sand itself. The model highlighted here is adapted from the Clay And Sand Model (CASM) developped by $\mathrm{Yu}$ [1] and the cementention model developped by Gens and Nova [2]. This approach, called Cemented Clay And Sand Model (CCASM), has succesfully been applied to cemented sands (see Rios et al. [3]).

The model is calibrated and validated with the use of standard tests realized in collaboration with INSA of Lyon. It is completed with the study of an uniaxial compression test observed by tomography. 3D FEM calculations should be able to predict the fracture surfaces initialization of the cemented sand, therefore the comparison with a non-homogeneous 3D strain field is particularly crucial.

\footnotetext{
${ }^{\star}$ e-mail: william.hilth@mines-paristech.fr
}

$\star \star$ e-mail: david.ryckelynck@mines-paristech.fr

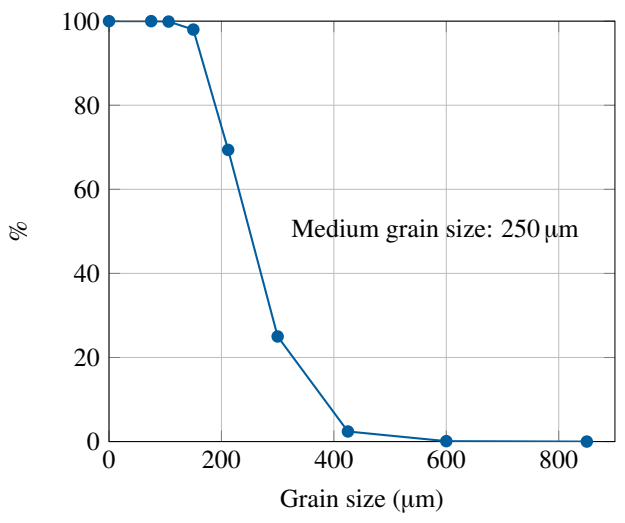

Figure 1. Grain size distribution

This study is focused on a polyurethane bonded silica sand (1\%wt. of resin). The grain size distribution is plotted in Figure 1.

\section{Model}

\subsection{Yield surface and plastic flow}

The constitutive elasto-plastic model, called Cemented Clay And Sand Model (CCASM), is the extension for cemented soils of the Clay And Sand Model developped by $\mathrm{Yu}$. The cementation is taken into account by the introduction of a scalar variable $b$ named bonding parameter. This 
variable and its evolution enable to model the intergranular bonding and its decay.

The bonding parameter amplifies the yield surface, assuming that its shape is the same as the yield surface of the unbonded sand. The amplification of the yield surface is controled by the isotropic compression and tensile yields:

$$
\begin{aligned}
p_{o} & =p_{c}(1+b) \\
p_{t} & =\alpha p_{c} b
\end{aligned}
$$

where $p_{c}$ is the isotropic compression yield for the uncemented sand and $\alpha$ a constant scalar parameter for the traction resistance. We assume here, for simplicity, a linear elasticity. The yield surface of the CCASM is:

$$
f\left(\sigma ; p_{c}, b\right)=\left(\frac{q}{M\left(p+p_{t}\right)}\right)^{n}+\frac{1}{\ln r} \ln \left(\frac{p+p_{t}}{p_{c}(1+b)+p_{t}}\right)
$$

where $p$ is the hydrostatic pressure, $q$ the equivalent von Mises stress and $M$ is the stress ratio $\eta=q /\left(p+p_{t}\right)$ at critical state. For $p \rightarrow-p_{t}$ we impose $f=0$.

In the critical state framework, the soil behavior is assumed to rely on the stress ratio:

- $\eta>M$ : contraction of the soil.

- $\eta=M$ : shearing can occur without any changes in stress or volume. In this case, the incremental volumetric plastic strain is zero.

- $\eta<M$ : dilatation of the soil.

The yields surfaces for the bonded and unbonded soil are plotted in Figure 2. For each configuration, the Critical State Line $(\eta=M)$ is plotted in black.

The expression of $f$ is the sum of two terms:

- $\left(\frac{q}{M\left(p+p_{t}\right)}\right)^{n}$ that controls the behavior of the core when sheared

- $\frac{1}{\ln r} \ln \left(\frac{p+p_{t}}{p_{c}(1+b)+p_{t}}\right)$ that controls the hydrostatic closure of the yield locus

$n$ and $r$ are constant coefficients that control the shape of the yield. Increasing $n$ tends to bring closer the yield locus to the critical state line and closes more abruptly the yield locus. The spacing ratio $r$ enables to modulate the intersection of the yield locus and the Critical State Line, hence controlling the softening/hardening transition of the model.

Rowe flow rule for bonded soil has been developped for this model, based on the ratio between the incremental plastic volumetric strain and the incremental plastic shear strain:

$$
\frac{\dot{\varepsilon}_{v}^{p}}{\dot{\varepsilon}_{s}^{p}}=\frac{9(M-\eta)}{9+3 M-2 M \eta}
$$

We note here that at the critical state, we have $\dot{\varepsilon}_{v}^{p}=0$ as stated before. Moreover, for $\eta>M, \dot{\varepsilon}_{v}^{p}<0$ inducing a dilatation of the soil (alternatively, for $\eta<M$, the soil contracts).

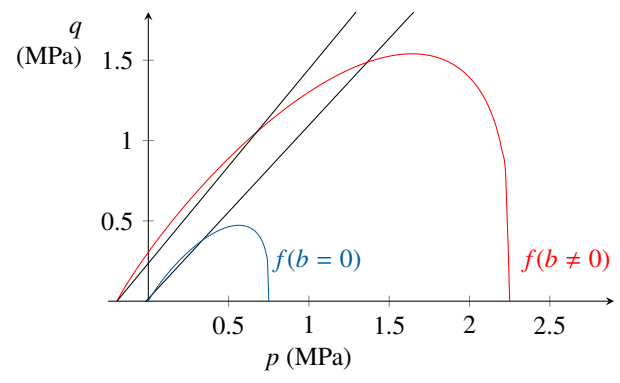

Figure 2. Yield surfaces in the $(p, q)$ plane

\subsection{Evolution of the parameters}

The model has a hardening variable $p_{c}$ and a plastic strain damage mesure, $h$, which ensures the irrecoverable decrease of $b$.

The evolution of the preconsolidation pressure is controled by the classical critical state rule:

$$
\frac{\dot{p_{c}}}{p_{c}}=\frac{v_{0}}{\lambda-\kappa} \dot{\varepsilon}_{v}^{p}
$$

where $\kappa$ and $\lambda$ are compressibility parameters of the uncemented sand and $v_{0}$ the initial specific volume. Combining this hardening rule and equation 2 , we can see that for a contraction of the soil $p_{c}$ decreases, leading to a softening of the bonded soil.

The bonding parameter $b$ decrease exponentially relatively to the plastic strain damage mesure evolution as:

$$
\begin{aligned}
& b=b_{0} \exp (-h) \\
& \dot{h}=h_{0}\left|\dot{\varepsilon}_{v}^{p}\right|+h_{1}\left|\dot{\varepsilon}_{s}^{p}\right|
\end{aligned}
$$

where $h_{0}$ and $h_{1}$ are two material parameters that control the sensibility of the material to plastic shear or volumetric strain.

Because of the decay of the intergranular bonding, we modeled the decrease of the effective stiffness modulus with a damage parameter $D$ that only depends on the evolution of $b$.

$$
\begin{aligned}
E & =E_{\text {ini }}(1-D) \\
D\left(b ; k_{0}, k_{1}\right) & =1-\frac{\sinh \left(k_{0}\left(b-k_{1}\right)\right)+\sinh \left(k_{1} k_{0}\right)}{\sinh \left(k_{0}\left(b_{0}-k_{1}\right)\right)+\sinh \left(k_{1} k_{0}\right)}
\end{aligned}
$$

where $k_{0}$ and $k_{1}$ are constant parameters and $b_{0}$ the initial value of $b$. The evolution of $D$ with respect to $b / b_{0}$ is plotted in Figure 3. We considered here that the damage of the media is only caused by the intergranular debonding. No damage mechanisms due to the sand itself (involving $p_{c}$ for instance) are considered. The damage law was calibrated using cycled uniaxial compression tests and assessing the loss of stiffness $\left(E / E_{i n i}\right)$ during plastic loading.

\section{Validation procedure}

\subsection{Idealized uniaxial compression test}

The first step in the calibration and validation of the CCASM is to model an idealized test and compare the 


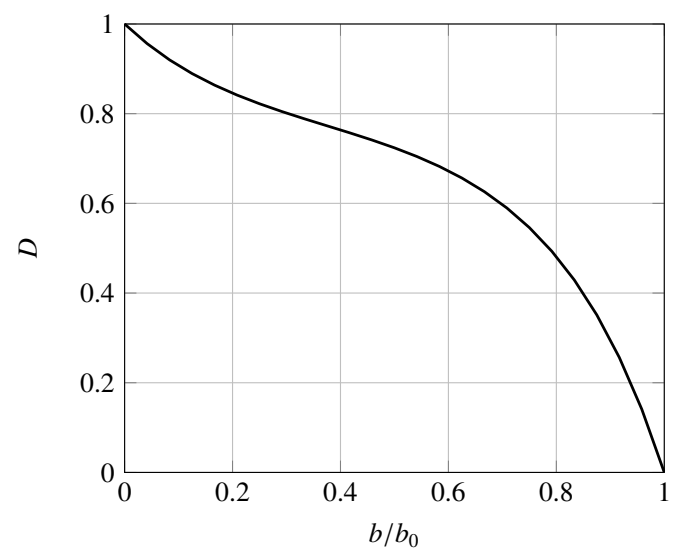

Figure 3. Evolution of $D$ with respect to $b / b_{0}$

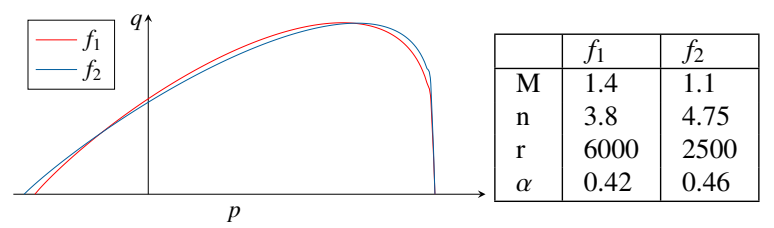

Figure 4. Redoundancy forecast in the parameters space

results with laboratory tests. The literature generally focuses on strain-stress curves or the evolution of plastic shear/volumetric strain during triaxial compression tests. This approach usually gives intervals of admissible parameters especially for the ones involved in the formulation of the yield surface. Given the uncertainties due to the tests, whether in the mesures themselves or in test repeatability (variability of the samples, initial inhomogeneity, ...), it is almost impossible to produce a single set of parameter values that can calibrate the model with only the data of standard tests. See for instance Figure 4 that shows two sets of parameters for the yield surface that lead to nearly the same yield locus.

In Figure 5, strain-stress curve for an uniaxial compression test are plotted. The model doesn't take into account for the pre-peak hardening as we considered the material elastic until the peak. This hypothesis tends to overevaluate the plastic strain. A modification of the CCASM with a multisurface approach can be imagined with one surface modeling the pre-peak hardening and the CCASM modeling the post-peak behavior (see for instance the Revised Modified Cam-clay model by Ami Saada et al. [6]). The damage law captures precisely the loss of stiffness through the loading. In Figure 6 the axial plastic strain and the damage parameter value taken from a cycled uniaxial compression test is compared to the FEM simulation.

Moreover, focusing only on these macroscopic curves doesn't allow to compare the fracture surfaces between tests and FEM simulations. For instance, for uniaxial compression test, the strain and stress fields during the postpeak domain are widely inhomogeneous as the localisation appears. This phenomenon makes troublesome to

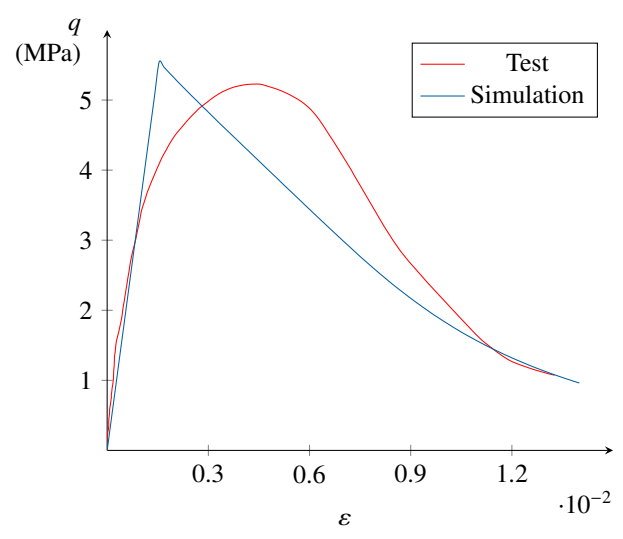

Figure 5. Comparison of strain-stress curves for uniaxial compression test

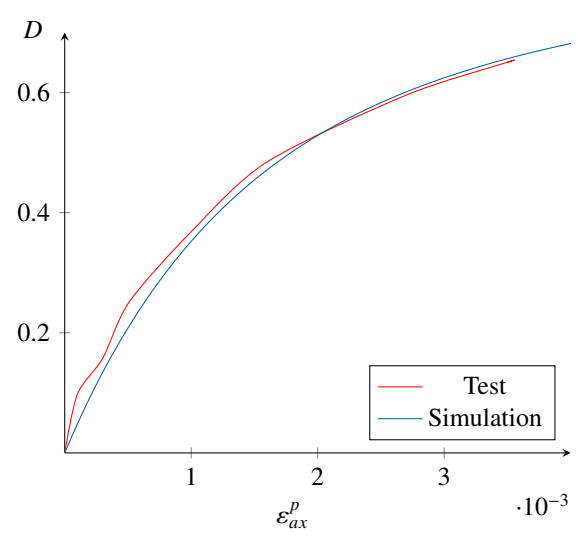

Figure 6. Evaluation of the damage law

evaluate the stress or strain with only the data of macroscopic curves. The parameters involved in the plastic flow or hardening rules are not obvious to calibrate. Thus, the constitutive model must be evaluated not only on the basis of standard laboratory tests but with the comparison of 3D inhomogeneous displacement fields.

\subsection{Uniaxial compression test studied in tomography}

As stated before, the model must predict correctly the fracture surfaces. Realistic displacement fields, taken from an uniaxial compression test on a cuboid observed in tomography, are considered as Dirichlet conditions for a FEM calculation. Unfortunately, these fields have missing experimental data because of the low correlations resolution for low displacement magnitudes. Moreover, the displacement fields are noisy. A method for reconstructing and filtering the data has been developped. This algorithm is based on the Gappy POD method [4, 5]. This method is:

1. Build a data base of $m$ points, or snapshots, where all the information is known at every time step

2. Build a proper order decomposition base $V$ of these snapshots 

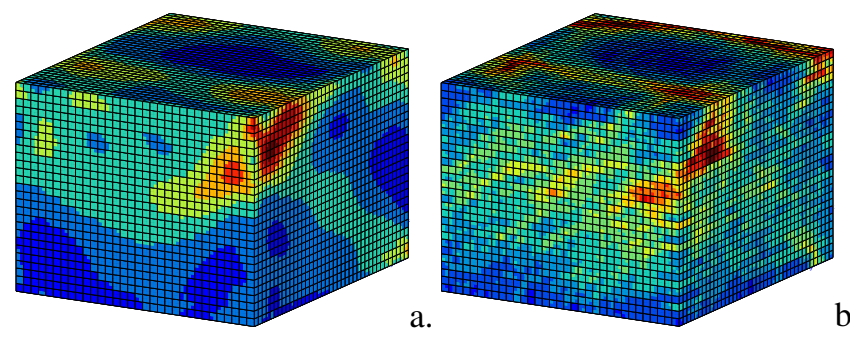

Figure 7. a. $\varepsilon_{s}$ of the model, b. $\varepsilon_{s}$ Gappy POD

3. At each points where the information is missing, apply the base $V$ in order to reconstruct the gappy field

4. Enrich the snapshots matrix with the new reconstructed points and continue the process until all the points are reconstructed

We used these reconstructed displacement fields as Dirichlet conditions at the top and the bottom of the cuboid sample. The simulation is performed until the post-peak domain. We compare the strain field calculated to the one determinated with the total displacement field applied to all the cuboid sample. Figure 7 shows the total shear strain, $\varepsilon_{s}$, for both approaches, as the fragmentation of the media is mostly due to shear strain. Figure 7 a. is displayed at a time step before Figure $7 \mathrm{~b}$..

Qualitatively, the simulation using the CCASM model forecasts the first slip in the top corner. A second conjugate shear band at $45^{\circ}$ is also predicted. This band is actually supposed to appear after. Figure $7 \mathrm{~b}$. shows the first time step of initialization of this band at a later stage of the loading. As pointed out before, the constitutive model tends to overevaluate the plastic strain. This explains the anticipation of this shear band.

The study of this FEM calculation has allowed a deeper comprehension of the parameters, especially those involved in the plastic flow. The resin-bonded sand possesses at the same time the caracteristics of a coesive material (via the intergranular bonding) and a frictional material (as a sand). Those different behaviors are modeled respectively by the evolution of $b$ (or $h$ ) and $p_{c}$. The study of the displacement field taken from the uniaxial compression test enabled to distinguish more accurately the relative importance of the decohesion or the frictional contraction of the soil. Both phenomena lead to a softening of the material that are fused in the macroscopic strain-stress curve.

\section{Conclusion}

The model was adapted and calibrated using a 3 steps method:

1. Adaptation of the Cemented Clay And Sand Model as described by Rios et al. (2016). The model was modified to the resin-bonded sand by considering a linear elasticity, changing the damage law and the plastic flow rule

2. Determination of intervals of correct parameters using macroscopic strain-stress curves taken from standard laboratory tests. These tests only gave qualitative informations on the fracture surfaces of the cemented sand subjected to various loading

3. Deeper study of realistic 3D displacement fields that enabled a better comprehension of the various parameters of the model, notably the impact of the decay of the intergranular bonding in the macroscopic behavior of the material.

The use of 3D FEM calculations is a relevant approach for calibrating and validating a model, especially for a softening material presenting strong inhomogeneities as studied here. This method is particularly suitable when the final aim is to anticipate the fracture modes of the media.

\section{Acknowledgements}

The authors would like to acknowledge the Agence Nationale pour la Recherche for their financial support for the FIMALIPO project.

\section{References}

[1] Yu H.-S., International Journal for Numerical and Analytical Methods in Geomechanics, 22, 621-653 (1998)

[2] Gens A. and Nova R., Geotechnical engineering of hard soils-soft rocks, 1, 485-494 (1993)

[3] Rios, S. and Ciantia, M. and Gonzalez, N. and Arroyo, M. and Viana da Fonseca, A., Computers and Geotechnics, 73, 100-108 (2016)

[4] Everson, R. and Sirovich, L., J. Opt. Soc. Am. A, 12,1657-1664 (1995)

[5] Carlberg, K. and Bou-Mosleh, C. and Farhat, C., International Journal for Numerical Methods in Engineering, 86, 155-181 (2011)

[6] Ami Saada, R. and Bonnet, G. and Bouvard, D., International Journal of Plasticity, 12, 273-294 (1996) 